\title{
Diagnostic ultrasound in sports medicine
}

Diagnostic ultrasound has in recent years been shown to be an extremely versatile and cost effective tool in the assessment of a wide range of musculoskeletal injuries and diseases. For superficial soft tissue structures such as the Achilles, patellar, rotator cuff, and plantar aponeurotic tendons, high resolution high frequency diagnostic ultrasound, in the hands of an appropriately trained sonologist, has a greater spatial and contrast resolution than magnetic resonance imaging. Until recently the ultrasound systems capable of producing the required image quality were the "top of the range" units costing around $£ 80000-$ $£ 120000$. It is important to realise, however, that a number of manufacturers' "flagship" systems, although expensive, are unable to provide good images for musculoskeletal ultrasound. Conversely, certain ultrasound equipment manufacturers have now started to develop dedicated portable musculoskeletal systems for a quarter to a third of this price. Dynamic Imaging Limited (Livingston, UK) for instance will be launching early in 1998 their application specific ultrasound systems - that is, the DIASUS - with one of these systems being specifically modified and targeted at the musculoskeletal ultrasound market. Also, interestingly it was recently announced by one large manufacturer from the USA that they will be releasing in the next two years a "pocket" unit which by the year 2005 may have a similar image quality to current high resolution systems. Like many things in life it is not necessarily size that counts!

Musculoskeletal ultrasound is able to be used to distinguish between chronic tendinitis, bursitis, and tenosynovitis/paratendonitis, to classify and quantify the extent of muscle injury, to evaluate joint dynamic and often static stabilisers, and to assess joint synovitis or fluid. It is also able to identify the precise site of superficial soft tissue inflammatory processes for localisation of therapeutic modalities and direct needle placement for aspiration or local anaesthetic/corticosteroid infiltration. Perhaps even more important in chronic repetitive minor injury overuse phenomena is the fact that musculoskeletal ultrasound is able to identify precise patterns of disease and therefore provide information about the underlying injury mechanism - that is, it is not only able to demonstrate the cause of an athlete's symptoms but the "cause of the cause". The fact that the imaging method is sensitive enough to demonstrate subclinical soft tissue degenerative changes and that there is no ionising radiation involved means that ultrasound may also be used as a screening procedure in an attempt to diagnose and correct underlying biomechanical faults before they even produce symptoms. It may also be used for follow up studies to assess the efficacy of therapeutic regimes.

The dedicated musculoskeletal ultrasound systems currently being developed are being aimed at the rheumatology, orthopaedic, sports medicine, and possibly physiotherapy markets. An increasing number of individuals involved in musculoskeletal imaging believe that, in five years time, when possible tendon, muscle, and superficial ligament soft tissue injuries are being considered, the clinical examination process will be "look, feel, move, ultrasound".

Therefore, if the most useful role for musculoskeletal ultrasound is in the clinic setting as an extension of physical examination, it would appear to be logical that either the ultrasound investigation should be performed by a radiologist with appropriate clinical experience in musculoskeletal injury and disease or a clinician/ physiotherapist with appropriate ultrasound training and experience. Indeed in many countries throughout the world-for example, Spain - most musculoskeletal ultrasound is performed by rheumatologists or sports physicians. The only important consideration should be that a patient/athlete receives the most cost effective high quality care possible in the current health-economic climate.

When considering medicolegal liability for therapeutic errors based on musculoskeletal ultrasound performed by non-radiologists, the situation is slightly unclear. Certainly many orthopaedic surgeons and rheumatologists perform their own arthrography, discography, etc, so presumably a legal precedent exists. Also, as musculoskeletal ultrasound in other EEC countries is performed regularly by non-radiologists, European Law must have a precedent. Perhaps anyone considering performing musculoskeletal ultrasound should, however, initially consult their Trust or insurance organisation about their liability. Again the important factor would seem to be the level of training and experience achieved.

In the diagnostic range, ultrasound itself is inherently safe. The health risks from ultrasound relate to misdiagnosis and not any radiation produced. Integral to the expansion of musculoskeletal ultrasound is therefore the quality of training provided in the use of the technique. Recent negotiations have taken place with a number of the manufacturers interested in expanding the musculoskeletal ultrasound market with regard to the incorporation of training and in-service clinical support within the equipment purchase price in order to help maintain appropriately high imaging standards. Optimisation of imaging standards would seem to be in everyone's interest, whether the imager/clinician, the manufacturer or, most importantly, the patient. Such considerations are important, as in diagnostic ultrasound, the correct scanning technique is just as important as a knowledge of anatomy and pathology and image interperation skills if diagnostic errors are to be avoided. As we enter the new millenium, if the expansion of musculoskeletal ultrasound is correctly controlled in terms of education and training, it could become one of the most valuable tools in the armamentarium of all involved in the prevention, assessment, and rehabilitation of sports injuries.

WAYNE W GIBBON

Visiting Professor in Sports Medicine, Leeds Metropolitan University, Director of Sports Medicine and Consultant Musculoskeletal Radiologist, United Leeds Teaching Hospitals NHS Trust 